\title{
Identification of $J A K 2$ (V617F) Mutation in Myeloproliferative Neoplasms by Using Allele Specific Polymerase Chain Reaction (AS-PCR)
}

\author{
Khin La Pyae Tun ${ }^{*}$, Aung Zaw Latt ${ }^{1}$, Win Pa Pa Naing1, San San Htwe1, Yamin Ko Ko1, \\ Win Win Mar' ${ }^{1}$, San Yu Hlaing ${ }^{1}$, Wai Wai Han ${ }^{1}$, Sein Win ${ }^{2}$ \\ ${ }^{1}$ Department of Medical Research, Yangon, Myanmar \\ ${ }^{2}$ Department of Clinical Haematology, Yangon General Hospital, Ministry of Health and Sports, Nay Pyi Taw, Myanmar \\ Email: *klapyaetun@gmail.com
}

How to cite this paper: Tun, K.L.P., Latt, A.Z., Naing, W.P.P., Htwe, S.S., Ko, Y.K., Mar, W.W., Hlaing, S.Y., Han, W.W. and Win, S. (2020) Identification of JAK2 (V617F) Mutation in Myeloproliferative Neoplasms by Using Allele Specific Polymerase Chain Reaction (AS-PCR). American Journal of Molecular Biology, 10, 273-282. https://doi.org/10.4236/ajmb.2020.104019

Received: February 29, 2020

Accepted: September 20, 2020

Published: September 23, 2020

Copyright $\odot 2020$ by author(s) and Scientific Research Publishing Inc. This work is licensed under the Creative Commons Attribution International License (CC BY 4.0).

http://creativecommons.org/licenses/by/4.0/

\begin{abstract}
Myeloproliferative neoplasms (MPNs) are a group of clonal haematopoietic stem cell disorders characterized by the proliferation of one or more myeloid cell lineages. According to WHO classification, the Janus associated kinase 2 (JAK2) V617F mutation is one of the major diagnostic criteria in BCR-ABL1 negative myeloproliferative neoplasms. The aim of this study is to detect the $J A K 2$ (V617F) mutation in patients with myeloproliferative neoplasms to get accurate diagnosis and proper management. A total of 90 clinically diagnosed MPN patients attending to Department of Clinical Haematology, Yangon General Hospital were enrolled in this study. The mean age was $53.4 \pm 14$ years which ranged from 16 to 81 years old and male and female ratio was 2.4:1. The identification of $J A K 2$ (V617F) point mutation was found to be positive in 44/90 MPN patients (48.9\%). According to MPN subtypes, the $J A K 2$ mutation positivity was found in 19 out of 46 polycythemia vera patients (41.3\%), 17 out of 25 essential thrombocythemia patients (68\%), 8 out of 15 primary myelofibrosis patients (53.3\%), 0 of 4 others myeloproliferative neoplasms (0\%). Confirmation of each of nine $J A K 2$ mutation positive and negative samples was done by Sanger sequencing. The arterial or venous thrombotic attack was found in 32/44 JAK2 mutation positive cases (72.7\%) and $12 / 44 J A K 2$ mutation negative cases (27.3\%). The association between thrombotic attack and presence of $J A K 2$ mutation was statistically significance with $p=0.000$. The diagnosis of myeloproliferative neoplasms mainly relies on the molecular genetics according to WHO classification. The Allele specific PCR reaction is sensitive, simple test and relatively cost-effective. There-
\end{abstract}


fore, the identification of $J A K 2$ (V617F) somatic point mutation by AS-PCR should be implemented as a routine diagnosis procedure for patients with chronic and suspected myeloproliferative neoplasms.

\section{Keywords}

Myeloproliferative Neoplasms, JAK2 (V617F), Allele-Specific PCR

\section{Background Information}

Myeloproliferative neoplasm (MPN), known as the former Myeloproliferative disorder (MPD), is a group of various chronic myeloid cancer: mainly consisting of the Philadelphia chromosome positive $(\mathrm{Ph}+)$ and Philadelphia chromosome negative $(\mathrm{Ph}-)$. For the $(\mathrm{Ph}-) \mathrm{MPN}$, there are polycythemia vera (PV), essential thrombocythemia (ET), primary myelofibrosis (PMF) and other rare myeloid disorders. The presentation of MPN is vague clinical symptoms but without proper medical treatment, it could be life-threatening if bleeding or thrombosis occur in vital organs. According to WHO (2008) classification, the Janus associated kinase 2 (JAK2) V617F mutation is the one of the major diagnostic criteria in ( $\mathrm{Ph}-)$ myeloproliferative neoplasm [1] [2] [3] [4] [5]. JAK2 V617F mutation is acquired somatic point mutation which results in a valine-to-phenylalanine substitution at position V617F in the pseudokinase domain of chromosome 9p which leads to constitutive activation and subsequently uncontrolled cell proliferation in the absence of growth factor. The mutation is somatic and has not been detected in any normal individuals or patients with reactive myeloproliferation, lymphoid disorders, or solid tumors [6] [7] [8] [9] [10]. This mutation is present in nearly all (97\%) of patients with PV, approximately $50 \%$ of each of those with ET and PMF, $20 \%$ of those with atypical MPNs, and $0 \%$ of those with chronic myelogenous leukemia (CML) [11]. There are several laboratory methods for detecting the $J A K 2$ (V617F) mutation, with varying assay sensitivity and specificity [12] [13] [14] [15]. AS-PCR and PCR-RFLP assays are useful screening tools for mutation of this nature as the two assays are easy and inexpensive to perform. But the disadvantages of these assays include post PCR processing is required which makes time consuming and risk contamination. AS-PCR has been claimed to have analytical sensitivity of between $0.01 \%$ and $3 \%$ while PCR-RFLP has sensitivity of around $5 \%$. Direct sequencing technique is not suitable for routine diagnosis laboratory as it is time-consuming and laborious. Studies have shown that sequencing has low detection sensitivity between 5\% and 20\% [16] [17] [18] [19] [20]. Therefore, we had been used the AS-PCR for identification of $J A K 2$ mutation in this study. In Myanmar, JAK2 mutation study in myeloproliferative neoplasms has not been well established yet. Therefore, this study aims to identify the $J A K 2$ mutation in patients with chronic and suspected MPN and detection of the mutation is helpful in differential diagnosis, prognosis, and predication of therapeutic response. 


\section{Material and Methods}

\subsection{Patients and Clinical Features}

This is the cross sectional descriptive study. The study was approved by Institutional Review Board of Department of Medical Research. We recruited the 90 patients diagnosed and suspected with Myeloproliferative Neoplasms attending to outpatient and in patients Department of Clinical Haematology, Yangon General Hospital from October 2018 to September 2019. All Written informed consent was obtained from every patient. The clinical features at diagnosis date in medical records were extracted to correlate with the mutation status, the information including red blood cell (RBC) count, haemoglobin $(\mathrm{Hb})$, haematicrit (Hct), mean corpuscular volume (MCV), white blood cell (WBC) count, platelet count and bleeding and thrombosis history.

\subsection{Specimen Processing}

$3 \mathrm{ml}$ of venous blood was taken under aseptic condition and collected with EDTA tube. The fresh whole blood was centrifuged and WBC from buffy coat layer was taken for DNA extraction which was done by QIA amp DNA minikit (Qiagen, Germany) following manufacturer's instructions. The samples were affirmed DNA validity by optical density measurement and then stored at $-20 \mathrm{C}$ before $J A K 2$ V617F examination.

\subsection{JAK2 V617F Examination}

The Allele-Specific PCR was done by using Forward wild type primer (FC) 5'-ATC TAT AGT CAT GCT GAA AGT AGG AGA AAG-3', Forward mutant primer (FM) 5'-AGC ATT TGG TTT TAA ATT ATG GAG TAT ATT-3' and Reverse primer (FR) 5'-CTG AAT AGT CCT ACA GTG TTT TCA GTT TCA-3' tocover the mutation point Val617Phe (Baxter et al.). The amplification reactions will be carried out in a $25 \mu \mathrm{l}$ total reaction volume by using a QIAGEN Top Taq PCR Master Mix with each of $0.6 \mathrm{ul}$ forward primer and $0.8 \mathrm{ul}$ reverse primer and $5 \mu \mathrm{l}$ of DNA extracted according to the manufacturer's protocol. The thermal profile of PCR is $95^{\circ} \mathrm{C}$ for $3 \mathrm{~min}$, followed by 35 cycles of $94^{\circ} \mathrm{C}$ for 30 secs, $54^{\circ} \mathrm{C}$ for $30 \mathrm{sec}$, and $72^{\circ} \mathrm{C}$ for $30 \mathrm{sec}$, and a final extension at $72^{\circ} \mathrm{C}$ for 5 min. After that, the product was applied onto $2 \%$ agarose gel for electrophoresis and undergone detection of product under UV light.

\subsection{Confirmation by Sanger Sequencing}

To validate the AS-PCR assay, each of nine $J A K 2$ positive and negative samples was analyzed by direct sequencing of the PCR product using Genetic Analyzer ABI 3100 (Applied Bio system).

\subsection{Statistical Analysis}

Statistical analysis was performed with IBM SPSS version 23. Descriptive statistics used to describe clinical, hematological characteristics and presence of $J A K 2$ 
mutation. Mean (SD) was used to summarize continuous variables and percentage was used to describe categorical variables. Appropriate test statistics (Independent $t$ test and ANOVA, Pearson chi-square test) were used to correlate the $J A K 2$ mutation and its clinico-haematological characteristics.

\section{Results}

\subsection{Clinical Features}

The mean age was $53.4 \pm 14$ years which ranged from 16 to 81 years old and male and female ratio was 2.4:1. Clinical features including age of diagnosis, bleeding, thrombotic attack such as CVA, DVT, smoking history and general hematological parameters are summarized in Table 1 . The data indicated that three parameters of red cell ( $\mathrm{Hb}, \mathrm{Hct}, \mathrm{RBC})$ in PV showed statistically significant difference to others: $\mathrm{Hb}, \mathrm{Hct}, \mathrm{RBC}$ values were higher compare to ET, PMF and MPNun $(p=0.000)$. The total WBC, neutrophil and platelet count showed increased in ET and PMF than PV and MPNun $(p=0.001, p=0.001, p$ $=0.000)$ respectively. The red cells parameters are significantly decreased in PMF comparing to PV and ET though total WBC and platelet count are increased.

\subsection{JAK2 V617F Mutation and Clinical Correlations}

The AS-PCR technique for $J A K 2 \mathrm{~V} 617 \mathrm{~F}$ examination is principally depended on the capture of two primers to the template: the outer wild-type and the inner mutant. The two nucleotide products different in size were observed: the $365 \mathrm{bp}$ band as the wild type and $203 \mathrm{bp}$ band as JAK2 V617F (Figure 1). Sequence analysis of $1849 \mathrm{G}>\mathrm{T}$ alteration was found in $J A K 2$ positive $\mathrm{PV}$ patient by Sanger sequencing, shown in Figure 2.

Table 1. Clinical data of study population.

\begin{tabular}{|c|c|c|c|c|c|c|}
\hline & Over all & PV & ET & PMF & MPN un & $p$-value \\
\hline Age & & $52.3 \pm$ & 59.44 & 48.3 & & \\
\hline $\mathrm{Hb}(\mathrm{g} / \mathrm{dl})$ & $14.6 \pm 4.4$ & $18+18$ & $121+23$ & $8.7 \pm 3.7$ & $14.4 \pm 1.6$ & $0.000^{\star} \mathrm{b}$ \\
\hline Hct (\%) & $44.9 \pm 13.7$ & $54.8 \pm 6.5$ & $37.9 \pm 8.7$ & $26.8 \pm 12.8$ & $42.5 \pm 4.4$ & $0.000^{\star} \mathrm{b}$ \\
\hline $\mathrm{RBC}(10 \times 96 / \mathrm{ul})$ & $5.7 \pm 1.6$ & $6.6 \pm 1.1$ & $5.3 \pm 1.4$ & $4.0 \pm 1.8$ & $5.2 \pm 0.9$ & $0.000^{\star} \mathrm{b}$ \\
\hline $\operatorname{MCV}(\mathrm{fl})$ & $77.9 \pm 13.3$ & $83.9 \pm 8.6$ & $72.5 \pm 13.6$ & $70.0 \pm 16.4$ & $82.9 \pm 8.9$ & $0.000^{*} b$ \\
\hline WBC $(10 \times 9 / L)$ & $17.5 \pm 14.4$ & $12.7 \pm 8.2$ & $21.5 \pm 15.7$ & $27.0 \pm 20.9$ & $7.7 \pm 4.9$ & $0.001^{\star} \mathrm{b}$ \\
\hline Neut $(10 \times 99 / \mathrm{L})$ & $13.0 \pm 12.7$ & $8.5 \pm 7.7$ & $16 \pm 13.7$ & $22.6 \pm 16.6$ & $4.0 \pm 2.6$ & $0.001^{\star} \mathrm{b}$ \\
\hline Plt $(10 \times 99 / \mathrm{L})$ & $697.6 \pm 599.1$ & $377.3 \pm 244.3$ & $1132.4 \pm 554.4$ & $1004 \pm 827.5$ & $240.0 \pm 177.7$ & $0.000^{\star} \mathrm{b}$ \\
\hline Thrombosis & $43 / 90(47.8 \%)$ & $24 / 45(55.8 \%)$ & $15 / 25(34.9)$ & $4 / 15(9.3 \%)$ & $0 / 4(0 \%)$ & $0.032^{\star} a$ \\
\hline Bleeding & $4 / 90(4.4 \%)$ & $0 / 45(0 \%)$ & $3 / 25(12 \%)$ & $1 / 15(6.7 \%)$ & $0 / 4(0 \%)$ & $0.079 a$ \\
\hline Smoking & $39 / 90(43.3 \%)$ & $24 / 45(53.3 \%)$ & $12 / 25(48.0 \%)$ & $2 / 15(13.3 \%)$ & $1 / 4(25 \%)$ & $0.033^{*} a$ \\
\hline
\end{tabular}

Haematological parameters in Mean \pm SD, Bleeding, thrombosis and smoking history in frequency/total cases. $\mathrm{a}=$ Person chi square test, $\mathrm{b}=$ one way Anova test. Hb (Haemoglobin), Hct (Haematocrit), MCV (Mean corpuscular volume), WBC (White blood cell count), Neut (Neutrophil count), Plt (platelet count). ${ }^{*}$ Statistical difference. 


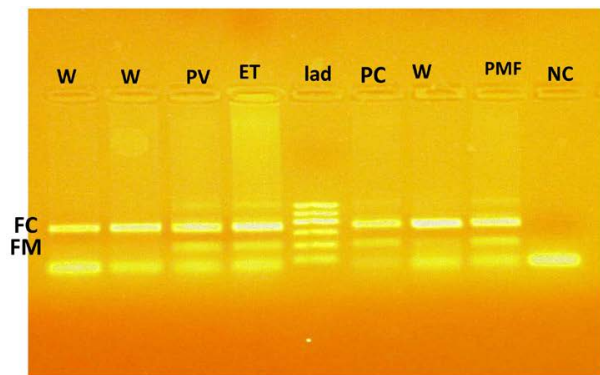

Fc-internal PCR control (364 bp), FM-mutant allele (203bp)

W-Wild type, PV-Polycythemia Vera, ET-

Essential Thrombocytosis, NC-Negative

Control $\left(\mathrm{H}_{2} \mathrm{O}\right)$, Lad- Ladder, PC-Positive Control,

PMF- Primary Myelofibrosis

Figure 1. Gel image showing AS-PCR.

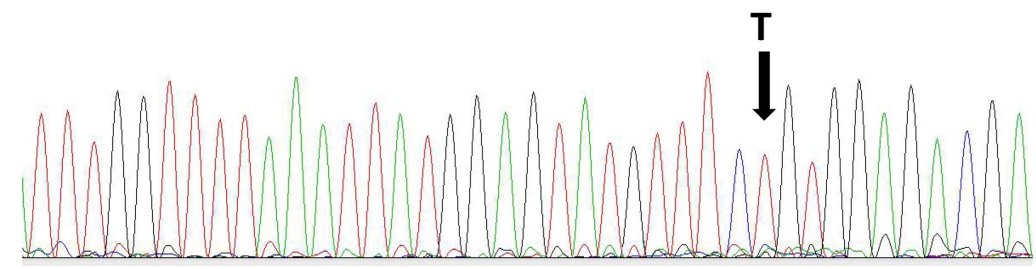

Figure 2. Sequence trace showing $\mathrm{G} \rightarrow \mathrm{T}$ mutation.

In this study the identification of $J A K 2 \mathrm{~V} 617 \mathrm{~F}$ point mutation was found to be positive in 44/90 MPN patients (48.9\%). According to MPN subtypes, the $J A K 2$ V617F mutation positivity was found in 19 out of 46 polycythemia vera patients (41.3\%), 17 out of 25 essential thrombocythemia patients (68\%), 8 out of primary myelofibrosis patients (53.3\%), 0 of 4 unclassified myeloproliferative neoplasms (0\%). The arterial or venous thrombotic attack was found in 32/44 JAK2 mutation positive cases (72.7\%) and $12 / 44 J A K 2$ mutation negative cases $(27.3 \%)$. The association between thrombotic attack and presence of $J A K 2$ mutation was statistically significance with $p=0.000$. The bleeding history was found only in 3 $(6.8 \%)$ of $J A K 2$ positive cases and not significant. Total of 15 mutation positive patients $(34.1 \%)$ has a history of smoking and $24(53.3 \%)$ of mutation negative patients has smoking history with statistically significance with $p=0.05$. The association between clinico-haematological findings and $J A K 2$ V617F mutation status were described in Table 2 . Those with mutation showed no significant difference in red cell parameters such as $\mathrm{Hb}$, Hct and RBC compared to wild type. The mutant group showed significant less MCV than wild type $(p=0.011)$. Total WBC, neutrophil count and platelet count were higher in mutant group than wild type with statistically significant $(p=0.000, p=0.000, p=0.017)$ respectively.

\section{Discussion}

The myeloproliferative disorders were classified as a spectrum of related disease in 1951 by Dameshek Discovery of $J A K 2$ V617F mutation has brought new insight to MPN, especially its contributions to the disease [2]. Detection of the $J A K 2 \mathrm{~V} 617 \mathrm{~F}$ mutation is important implications for classification, diagnosis and 
Table 2. Association between clinico-hematological findings and $J A K 2$ mutation status.

\begin{tabular}{cccc}
\hline & $\begin{array}{c}\text { JAK2 V617F mutation } \\
\text { Positive (Mutant type) }\end{array}$ & $\begin{array}{c}J A K 2 \text { V617F mutation } \\
\text { Negative (Wild type) }\end{array}$ & $p$-value \\
\hline Age & $56.5 \pm 11.7$ & $50.5 \pm 15.5$ & $0.043^{\star} \mathrm{b}$ \\
$\mathrm{Hb}(\mathrm{g} / \mathrm{dl})$ & $14.4 \pm 4$ & $14.9 \pm 4.6$ & $0.56 \mathrm{~b}$ \\
$\mathrm{Hct}(\%)$ & $44.8 \pm 13.4$ & $44.1 \pm 13.2$ & $0.906 \mathrm{~b}$ \\
$\mathrm{RBC}(10 \times 6 / \mathrm{ul})$ & $6 \pm 1.6$ & $5.5 \pm 1.6$ & $0.198 \mathrm{~b}$ \\
$\mathrm{MCV}(\mathrm{fl})$ & $74 \pm 15.2$ & $81.5 \pm 10$ & $0.011^{\star} \mathrm{b}$ \\
$\mathrm{WBC}(10 \times 9 / \mathrm{L})$ & $24 \pm 16.4$ & $11 \pm 8.2$ & $0.000^{*} \mathrm{~b}$ \\
$\mathrm{Neut}(10 \times 9 / \mathrm{L})$ & $19.2 \pm 14.7$ & $7.3 \pm 6.7$ & $0.000^{\star} \mathrm{b}$ \\
$\mathrm{Plt}(10 \times 9 / \mathrm{L})$ & $851.1 \pm 529.5$ & $526 \pm 420.2$ & $0.017^{\star} \mathrm{b}$ \\
Thrombosis & $32 / 44(72.7 \%)$ & $11 / 46(24.4 \%)$ & $0.000^{*} \mathrm{a}$ \\
Bleeding & $3 / 44(6.8 \%)$ & $1 / 46(2.2 \%)$ & $0.361 \mathrm{a}$ \\
Smoking & $15 / 44(34.09 \%)$ & $24 / 46(52.17 \%)$ & $0.053^{*} \mathrm{a}$ \\
\hline
\end{tabular}

Hematological parameters in Mean \pm SD, Bleeding, thrombosis and smoking history described in frequency/total cases. $\mathrm{a}=$ Person chi square test, $\mathrm{b}=$ Independent $\mathrm{t}$ test. Hb (Hemoglobin), Hct (Haematocrit), MCV (Mean corpuscular volume), WBC (White blood cell count), Neut (Neutrophil count), Plt (Platelet count). ${ }^{\star}$ Statistical difference.

treatment of these disease and provide insight into their pathogenesis [21]. However, information in Asian especially in Myanmar has not been well studied enough. Our study described the MPN patients emphasizing prevalence and clinical correlation of the mutation. The clinical correlation of $J A K 2$ is a topic to be discussed. The mean age of MPN patients was $53.4 \pm 14$ years which ranged from 16 to 81 years old and male and female ratio was 2.4:1. The youngest age of the $J A K 2 \mathrm{~V} 617 \mathrm{~F}$ mutation positive patients was 35 years old in our study. The prevalence of $J A K 2$ V617F mutation is reported in many studies worldwide, approximately $80 \%-90 \%$ in PV, $50 \%-60 \%$ in ET, $40 \%-50 \%$ in PMF and $20 \%$ in unclassifiable MPN [4] [11] Studies in Asia, it was $80 \%-90 \%$ in PV, $50 \%-60 \%$ in ET, 30\% - 50\% in PMF [22] [23] [24]. The data reported are close to both regional and global data. This could be assumed that ethnicity has less effect to $J A K 2 \mathrm{~V} 617 \mathrm{~F}$ prevalence. In our study, the identification of $J A K 2 \mathrm{~V} 617 \mathrm{~F}$ point mutation was found to be positive in 44/90 MPN patients (48.9\%) with 19 out of 46 polycythemia vera patients (41.3\%), 17 out of 25 essential thrombocythemia patients (68\%), 8 out of 15 primary myelofibrosis patients (53.3\%), 0 of 4 unclassified myeloproliferative neoplasms (0\%). Although the prevalence of mutation in ET and PMF are not quite different, the mutation prevalence was found to be lower in PV compare to other studies. It may be due to secondary polycythemia in which smoking and other disorders that affect the erythropoietin production. We also found that two parameters of red blood cells $(\mathrm{Hb}, \mathrm{Hct})$ values showed no different between two groups and also mutant group had lower MCV than wild type with $p=0.011$. It may possibly be caused by iron deficiency which is common in Myanmar. Moreover, study on a large scale is required for more 
precision to cover the differences in mutation incidence of MPN. To distinguish myeloproliferative neoplasms from reactive condition, particularly secondary thrombocytosis and erythrocytosis, can be difficult and so detection of the V617F mutation could become a widely use diagnostic test with less invasive and cost effective. So, our study highlighted the important of $J A K 2$ examination to distinguish between primary and secondary polycythemia vera. In the study of mutation screening for $J A K 2$ V617F, Tefferi et al. (2006) reported that $J A K 2$ V617F mutation analysis can be used to help screen individuals with polycythemia and that this may reduce the need for further investigations, such as red cell mass and bone marrow biopsy [25]. In our study, most of the MPN patients were found to have the history of thrombosis and vascular complication such as CVA, fainting attack, numbness and tingling of the limbs and DVT. The arterial or venous thrombotic attack was found in 32/44 JAK2 mutation positive cases (72.7\%) and 12/44 JAK2 mutation negative cases (27.3\%). The association between thrombotic attack and presence of $J A K 2$ mutation was statistically significance with $p=0.000$. The finding was consistent with the others studies. But only 3 out of 44 mutation positive had bleeding history. The history of smoking was found to be higher in wild type group than mutant group with statistically significant $(p=0.053)$. Therefore, our finding emphasize that smoking might be one of the major causes of secondary polycythemia in patients with suspected MPNs. Clinical, biologic, and pathologic evidence indicates that ET, PV, and PMF are related disorders, and about $15 \%$ of patients with ET and $20 \%$ of those with PV experience progression to PMF and transform to acute leukemia in later stage [26]. In 2007 study, Barosis $\mathrm{G}$ et al. found that the strongest influence of the mutant genotype was on the risk of leukaemic transformation which was 5.2 times higher than that in patients whose disease lacked the mutation [27]. Thus, $J A K 2$ mutation should be considered as a prognostic risk factor independent from conventional predictor in myelofibrosis such as age, $\mathrm{Hb}$ level and WBC count. The $J A K 2$ V617F mutation has been detected in progenitors and myeloid cells including cells with hematopoietic stem cells, common myeloid progenitor and megakaryocyte-erythroid progenitor's phenotype as well as colony forming cells and more mature progenies, such as neutrophils and platelets [9] [10] but has not been reported in T or B lymphocytes [28]. The Medical Research Council Primary Thrombocythemia-1 Trial studied the effect of JAK2 V617F mutation on treatment outcome in patients with $\mathrm{ET}$ and $\mathrm{PV}$, demonstrating that $J A K 2$ V617F mutation-positive patients were much more sensitive to hydroxyurea, but not to anagrelide, than those without the JAK2 V617F mutation [29]. Identification of the Val617Phe mutation will also stimulate clinical development of small molecule inhibitors of $J A K 2$, which could provide novel approach to treatment. Recently, specific inhibitors of $J A K 2$ have been developed and employed in the treatment of $J A K 2$-mutated MPN [30].

\section{Conclusion}

Our study reported that $J A K 2$ V617F mutation status in patients with myelopro- 
lierative neoplasm in Myanmar. The mutant group displayed higher hematological parameters than wild type referring to contribution of the mutation of disease. Nevertheless, the frequency of mutation in polycythemia is lower in our study's highlight about the fact that the presence of $J A K 2$ V617F mutation is considered an important criterion for the exclusion of secondary/reactive from clonal disorders.

\section{Acknowledgements}

We would like to express our gratitude to Director General and Board of Directors, Department of Medical Research for their permission to conduct this study. We are also grateful to all patients for their willing cooperation.

\section{Conflicts of Interest}

The authors declare no conflicts of interest regarding the publication of this paper.

\section{References}

[1] Jaffe, E.S., Harris, N.L., Stein, H. and Vardiman, J.W. (2001) World Health Organization Classification of Tumors of Hematopoietic and Lymphoid Tissues. IARC Press, Lyon, 1-351.

[2] Dameshek, W. (1951) Some Speculations on the Myeloproliferative Syndromes. Blood, 6, 372-375. https://doi.org/10.1182/blood.V6.4.372.372

[3] Tefferi, A. and Vardiman, J.W. (2008) Classification and Diagnosis of Myeloproliferative Neoplasm: The 2008 WHO Diagnostic Criteria and Point of Care Algorithms. Leukemia, 22, 14-22. https://doi.org/10.1038/sj.leu.2404955

[4] Baxter, E.J., Scott, L.M., Campbell, P.J., et al. (2005) Acquired Mutation of the Tyrosine Kinase JAK2 in Human Myeloproliferative Disorders. The Lancet, 365, 1054-1061. https://doi.org/10.1016/S0140-6736(05)71142-9

[5] Vardiman, J.W., Thiele, J., Arber, D.A., Brunning, R.D., Borowit, Z.M.J., Porwit, A., et al. (2009) The 2008 Revision of the World Health Organization (WHO) Classification of Myeloid Neoplasm and Acute Leukaemia: Rationale and Important Changes. Blood, 114, 937-951. https://www.nejm.org/doi/full/10.1056/NEJMoa051113

[6] Kralovics, R., Passamonti, F., Buser, A.S., et al. (2005) A Gain-of-Function Mutation of JAK2 in Myeloproliferative Disorders. The New England Journal of Medicine, 352, 1779-1790. https://www.nejm.org/doi/full/10.1056/NEJMoa051113 https://doi.org/10.1056/NEJMoa051113

[7] James, C., Ugo, V., Le Couedic, J.P., et al. (2005) A Unique Clonal JAK2 Mutation Leading to Constitutive Signaling Causes Polycythaemiavera. Nature, 434, 1144-1148. https://doi.org/10.1038/nature03546

[8] Levine, R.L., Wadleigh, M., Cools, J., et al. (2005) Activating Mutation in the Tyrosine Kinase JAK2 in Polycythemia Vera, Essential Thrombocythemia, and Myelofibrosis with Myeloid Metaplasia. Cancer Cell, 7, 387-397.

[9] Jamieson, C.H., Gotlib, J., Durocher, J.A., Chao, M.P., Mariappan, M.R., Lay, M., Jones, C., et al. (2006) The JAK2 V617F Mutation Occurs in Hematopoietic Stem Cells in Polycythemia Vera and Predisposes toward Erythroid Differentiation. Proceedings of the National Academy of Sciences of the United States of America, 103, 
6224-6229. https://doi.org/10.1073/pnas.0601462103

[10] Kiladjian, J., Elkassar, N., Cassinat, B., et al. (2006) Essential Thrombocythemias without V617F JAK2 Mutation Are Clonal Hematopoietic Stem Cell Disorders. Leukemia, 20, 1181-1183. https://doi.org/10.1038/sj.leu.2404214

[11] Jones, A.V., Kreil, S., Zoi, K., Waghorn, K., Curtis, C., Zhang, L., Score, J., Seear, R., Chase, A.J., Grand, F.H., White, H., Zoi, C., Loukopoulos, D., Terpos, E., Vervessou, E.C., Schultheis, B., Emig, M., Ernst, T., Lengfelder, E., Hehlmann, R., Hochhaus, A., Oscier, D., Silver, R.T., Reiter, A. and Cross, N.C.P. (2005) Widespread Occurrence of the JAK2 V617F Mutation in Chronic Myeloproliferative Disorders. Blood, 106, 2162-2168. https://doi.org/10.1182/blood-2005-03-1320

[12] Jones, A.V., Silver, R.T., Waghorn, K., et al. (2006) Minimal Molecular Response in Polycythemia Vera Patients Treated with Imatinib or Interferon Alpha. Blood, 107, 3339-3341. https://doi.org/10.1182/blood-2005-09-3917

[13] James, C., Delhommeau, F., Marzac, C., et al. (2006) Detection of JAK2 V617F as a First Intention Diagnostic Test for Erythrocytosis. Leukemia, 20, 350-353. https://doi.org/10.1038/sj.leu.2404069

[14] Mcclure, R., Mai, M. and Lasho, T. (2006) Validation of Two Clinically Useful Assays for Evaluation of JAK2 V617F Mutation in Chronic Myeloproliferative Disorders. Leukemia, 20, 168-171. https://doi.org/10.1038/sj.leu.2404007

[15] Campbell, P.J., Scott, L.M., Baxter, E.J., Bench, A.J., Green, A.R. and Erber, W.N. (2006) Methods for Detection of the JAK2 V617F Mutation in Human Myeloproliferative Disorders. Methods in Molecular Medicine, 125, 253-264. https://doi.org/10.1385/1-59745-017-0:253

[16] Grenier, T.C. (2006) Diagnostics Assays for the JAK2 V617F Mutation in Chronic Myeloproliferative Disorders. American Journal of Clinical Pathology, 125, 651-653. https://doi.org/10.1309/NXXTGRCXD0TMA3C2

[17] Lay, M., Mariappan, R., Gotlib, J., Dietz, L., Sebastian, S., Schrijver, I. and Zehnder, J.L. (2006) Detection of the JAK2 V617F Mutation by LightCycler PCR and Probe Dissociation Analysis. The Journal of Molecular Diagnostics, 8, 330-334.

[18] Steensma D.P. (2006) JAK2 V617F in Myeloid Disorders: Molecular Diagnostic Techniques and Their Clinical Utility: A Paper from the 2005 William Beaumont Hospital Symposium on Molecular Pathology. The Journal of Molecular Diagnostics, 8, 397-526. https://doi.org/10.2353/jmoldx.2006.060007

[19] Kannism, S., Thongnoppakhun, W. and Auewarakul, C.U. (2009) Two-Round Allele Specific Polymerase Chain Reaction: A Simple and Highly Sensitive Method for JAK2 V617 F Mutation Detection. Clinica Chimica Acta, 401, 148-151. https://doi.org/10.1016/j.cca.2008.12.010

[20] Frantz, C., Sekora, D.M., Henley, D.C., Huang, C.-K., Pan, Q., Quiglay, N.B., Gorman, E., Hubbard, R.A. and Mirzo, I. (2007) Comparative Evaluation of Three JAK2 V617F Mutation Detection Methods. American Journal of Clinical Pathology, 128, 865-874. https://doi.org/10.1309/LW7Q3739RBRMBXXP

[21] Vainchenker, W. and Constantinescu, S.N. (2005) A Unique Activating Mutation in JAK2 (V617F) Is at the Origin of Polycythemia Vera and Allows a New Classification of Myeloproliferative Diseases. Hematology, American Society of Hematology Education Program, 2005, 195-200.

https://doi.org/10.1182/asheducation-2005.1.195

[22] Lieu, C.H., Wu, H.S., Hon, Y.C., Tsai, W.H., Yang, C.F., Wang, C.C., Lin, Y.C., Shih, C.H. and Hsu, H.C. (2008) Prevalence of the JAK2-V617F Mutation in Taiwanese Patients with Chronic Myeloproliferative Disorders. Internal Medicine 
Journal, 38, 422-426. https://doi.org/10.1111/j.1445-5994.2007.01589.x

[23] Kim, J.T., Cho, Y.G., Choi, S.I., Lee, Y.J., Kim, H.R., Jang, S.J., et al. (2010) JAK2 V617F and Exon 12 Genetic Variations in Korean Patients with $B C R / A B L 1$ Negative Myeloproliferative Neoplamsms. Korean Journal of Laboratory Medicine, 30, 567-574. https://doi.org/10.3343/kjlm.2010.30.6.567

[24] Wong, G.C., Kam, G.L. and Koay, E.S. (2011) JAK2 Mutations in Asian Patients with Essential Thrombocythaemia. Internal Medicine Journal, 41, 191-196. https://doi.org/10.1111/j.1445-5994.2010.02199.x

[25] Tefferi, A. and Pardanani, A. (2006) Mutation Screening for JAK2 ${ }^{\mathrm{V} 617 \mathrm{~F}}$ : When to Order the Test and How to Interpret the Results. Leukemia Research, 30, 739-744. https://doi.org/10.1016/j.leukres.2006.01.004

[26] Spivak, J.L. (2002) Polycythemia Vera: Myths, Mechanisms, and Management. Blood, 100, 4272-4290. https://doi.org/10.1182/blood-2001-12-0349

[27] Barosi, G., Bergamaschi, G., Marchetti, M., et al. (2007) JAK2 V617F Mutational Status Predict Progression to Large Splenomegaly and Leukaemic Transformation in Primary Myelofibrosis. Blood, 110, 4030-4036.

https://doi.org/10.1182/blood-2007-07-099184

[28] Lasho, T.L., Mesa, R., Gilliland, D.G. and Tefferi, A. (2005) Mutation Studies in $\mathrm{CD}^{+}, \mathrm{CD}_{19}{ }^{+}$and $\mathrm{CD} 34^{+}$Cell Fractions in Myeloproliferative Disorders with Homozygous $J A K 2^{\mathrm{V} 617 \mathrm{~F}}$ in Granulocytes. British Journal of Haematology, 130, 797-799. https://doi.org/10.1111/j.1365-2141.2005.05682.x

[29] Campbell, P.J., Scott, L.M., Buck, G., Wheatley, K., East, C.L., Marsden, J.T., Duffy, A., et al. (2005) Definition of Subtypes of Essential Thrombocythaemia and Relation to Polycythaemia Vera Based on JAK2 V617F Mutation Status: A Prospective Study. The Lancet, 366, 1945-1953. https://doi.org/10.1016/S0140-6736(05)67785-9

[30] Rosenthal, A. and Mesa, R.A. (2014) Janus Kinase Inhibitors for the Treatment of Myeloproliferative Neoplasms. Expert Opinion on Pharmacotherapy, 15, 1265-1276. https://doi.org/10.1517/14656566.2014.913024 